\title{
URTICARIA NEONATORUM (ERYTHEMA TOXICUM NEONATORUM)
}

\author{
BY \\ H. V. L. FINLAY and J. P. BOUND \\ From the Paediatric Unit, Hillingdon Hospital, Middlesex
}

(RECEIVED FOR PLBLICATION MAY 4, 1953)

A transient, urticaria-like rash in the first few days of the neonatal period is common and is familiar to the midwife as a trivial 'heat-rash'. Smellie (1752) described the condition in these words:

'The body of the child is sometimes covered all over with little red spots, called the red gum, and commonly proceeding from the costiveness of the child, when the Meconium hath not been sufficiently purged off at first.' He later (1764) mentioned one of his cases, 'A child, about three days after delivery, struck out all over the body with small red eruptions; which in London the nurses call the red-gum; but in Scotland is termed the hives.'

Several references can be found in medical books of the early nineteenth century. For example, Dewees (1826) wrote the following account of Strophulus Intertinctus, or Red Gum:

'This complaint is confined to early infancy, and especially to "the month", as it is called. Very few children escape this complaint; and most nurses are fond of seeing it-so much so indeed, and so inevitable and useful do they consider its presence, that should any indisposition befall the child, and this eruption not have possession of the skin, it is at once attributed to the absence of the gum. With a view then to invite its appearance, the child is kept unusually warm, and some stimulating tea is given it, such as of sweet marjoram, saffron, catmint, etc., and after having been thus disciplined for a longer or shorter period, the poor child is but too frequently loaded with a heavy crop of "Red Gum". This eruption, however, seems connected in some way or other with a derangement of the stomach and bowels ... This disease, under ordinary circumstances, requires little or no medical treatment.'

Evanson and Maunsell (1836) stated:

'Almost every infant is affected three or four days after birth, with an eruption of papulae. ... In its ordinary form this consists of a few red pimples, appearing in greatest number upon the face, neck, and hands, and interspersed with diffused red patches. . . The ordinary red gum of infancy can scarcely be called a disease. It arises, probably, from the slight irritation attendant upon the new circumstances under which the skin and mucous membranes are placed.'
According to Hardaway (1889), this rash had been termed "erythema papulosum of the new-born, or erythema neonatorum". He noted that its occurrence had occasionally given rise to much confusion in diagnosis, that the mucous membranes were unaffected, and that there was no evidence of systemic reaction.

To the same condition, Leiner (1912) gave the name 'erythema neonatorum toxicum'. He differentiated a simple erythematous type and a papular type, providing a comprehensive clinical description of each. In an important paper Mayerhofer and Lypolt-Krajnović (1927) described the rash as occurring in $46 \%$ of 1,115 newborn infants and noted that it appeared in the first four days of life in $96 \%$ of those affected. Salomonsen (1952) referred to the rash as 'allergic exanthem'.

The purpose of this paper is to draw attention to some features of the condition which have interested us, and to encourage further investigation into its cause. The following clinical observations are based on a series of cases of urticaria neonatorum seen by us in the Maternity Unit of this hospital over the two and a half-year period from August 15, 1950, to February 15, 1953.

\section{Clinical Features}

Urticaria neonatorum is seen in two forms: (1) a simple type and (2) a pustular type. In the words of Leiner, 'both these types may appear simultaneously in the same individual or they may appear separately. But one finds almost regularly the transition from one type to the other.'

As a rule the affected babies are otherwise healthy with no constitutional disturbance, such as fever or gastro-intestinal upset. Itching of the skin, indicated by fretfulness, is not evident. A moderate eosinophilia in the peripheral blood is often found.

The Simple Type. This type of urticaria neonatorum consists of small, blotchy, ill-defined areas of bright erythema, which may vary considerably in size. In the centre of each lesion is a white or yellow 


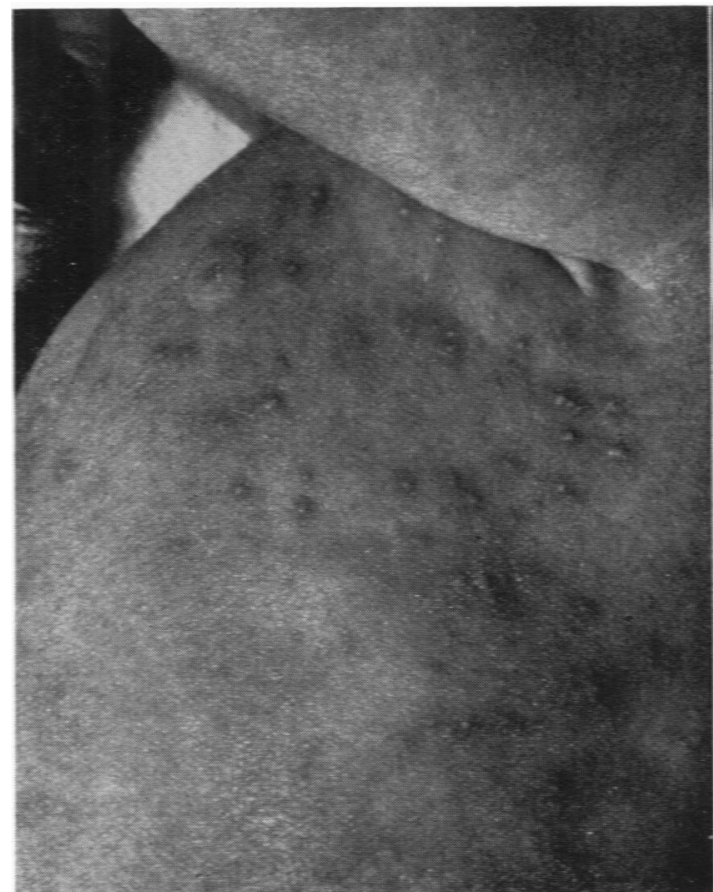

Fig. 1.-Typical non-infected pustular urticaria neonatorum affecting the axilla of an infant aged 3 days. (Reproduced by permission of the Editor, British Medical Journal.)

wheal, best seen by stretching the surrounding skin. These wheals can be easily felt on running a finger lightly over the affected areas: they are seldom much larger than $2-3 \mathrm{~mm}$. in diameter, and never attain the size found in the urticaria of older children.

The urticarial lesions are scattered irregularly over the body. Especially liable to be affected are the back, the buttocks and the extensor aspects of the arms. There may be fewer than half a dozen lesions in all at one time, or large confluent areas may occur, giving the skin a granular surface. The whole character of the rash may alter, even in a few hours. In all instances the rash fades rapidly over $\mathbf{2 4}$ hours, so that, usually when $\mathbf{4 8}$ hours have elapsed, there is little to be seen. Once the condition has cleared, recurrences are not seen as a rule while the infant remains in hospital. In one of our cases there was a recurrence of the rash, coinciding with the beginning of complementary feeding at the age of 7 days.

The Pustular Type. In some cases a varying number of the simple lesions develop rapidly into white or yellow 'pustules', owing to the intense local outpouring of eosinophil leucocytes in the centre of each lesion. We have so far encountered a clear

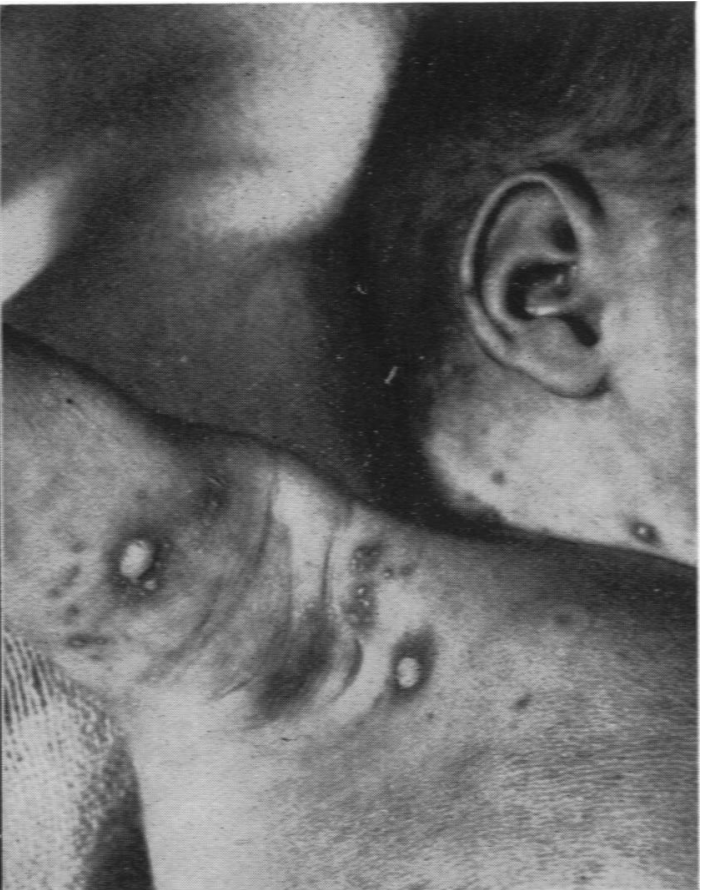

FKG. 2.-Staphylococcal pyodermia, for comparison, affecting the axilla of an infant aged 3 days.

vesicular stage in the evolution of the rash in only two instances. Each pustule is often surrounded by only a very narrow red base, but there may be no local skin reaction at all. Unless secondarily infected, these lesions never progress to the bullous formation often seen in staphylococcal pyodermia (Fig. 2).

Tending to develop in lesions in the body flexures, these pustules are found most frequently in the skin folds at both sides of the neck. Other favourite sites are the scalp around the ears, the axillae (Fig. 1) and the flexor aspect of the elbows. The pustular lesions may become very profuse, but many cases never show more than a few. Typical simple urticaria neonatorum lesions can usually be found elsewhere on the body.

Medical advice is usually sought when the rash assumes a frankly pustular appearance, but fortunately the eosinophil pustules rapidly dry up within one or two days without treatment, leaving small crusts which soon fall off. The importance of this pustular form of urticaria neonatorum lies in its very close resemblance at times to the pustular rash of true staphylococcal pyodermia.

Secondarily Infected Pustular Type. On several 


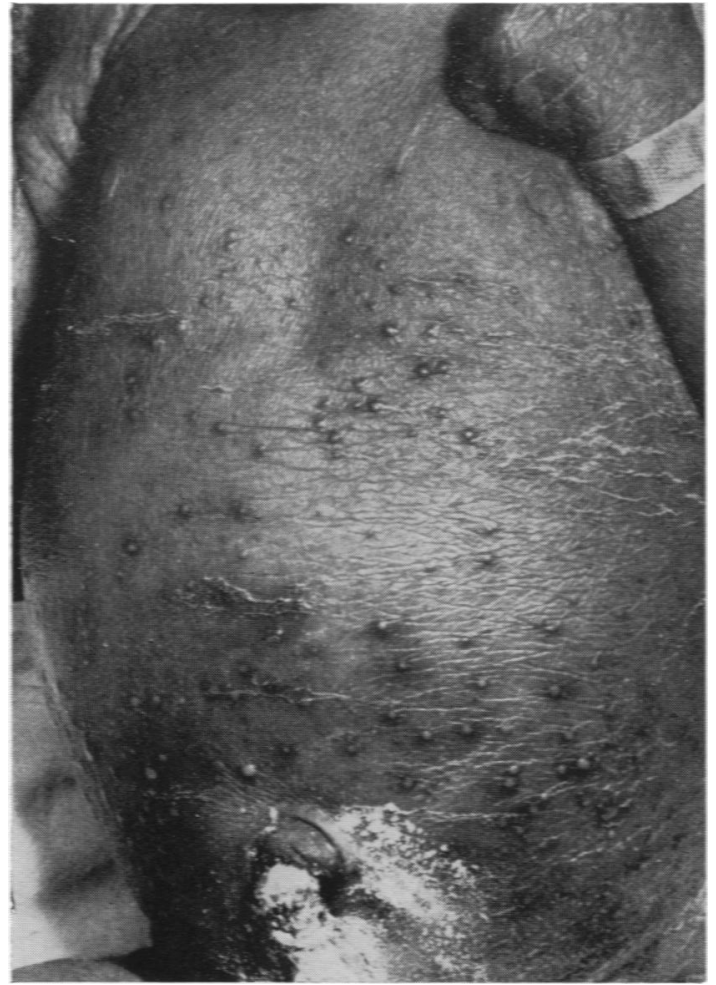

Fig. 3.-Pustular urticaria neonatorum, with earty secondary infection, in an infant aged 3 days.

occasions (five cases in the present series) lesions of pustular urticaria neonatorum have later become secondarily infected by coagulase-positive staphylococci, often in association with conjunctivitis, rhinitis or omphalitis, due to the same organism (Fig. 3). When this occurs the lesions persist longer and tend to increase in size. It is of interest that many lesions may become infected at once.

\section{Incidence}

The disorder is probably exceedingly common. Indeed, Salomonsen (1952) referring to all types, stated that allergic exanthem occurs 'on the third or fourth day in about $50 \%$ of all babies, if the mildest forms of the condition are included. During the past three or four years. our interest in urticaria neonatorum has stimulated the nursing staff to an increasing awareness of its occurrence. We have no information as to its frequency in domiciliary practice.

A total of 4,917 infants were born in the Unit during the period of observation: many mild examples of the simple type of urticaria neonatorum were encountered: 25 cases of the pustular type were seen.

Urticaria neonatorum is practically confined to the first week of life and is first noticed by the midwife most frequently at the age of 2 days. Among our 25 cases of the pustular type, the age of onset was 2 days in 11 , while $23(92 \%)$ of the cases occurred within the first four days of life. This contrasts sharply with staphylococcal pyodermia, which is very uncommon during the first three days of life and, in our experience, is encountered most frequently at the age of 5 days (Fig. 4).

All our cases have been entirely breast-fed at the first onset of the rash. There did not appear to be any particular seasonal incidence. In three of our cases of urticaria neonatorum there was a history of one or more siblings being similarly affected in the neonatal period.

\section{Diagnosis}

The diagnosis of simple urticaria neonatorum presents no difficulties, the central wheal in each lesion being the diagnostic feature. The wheals can

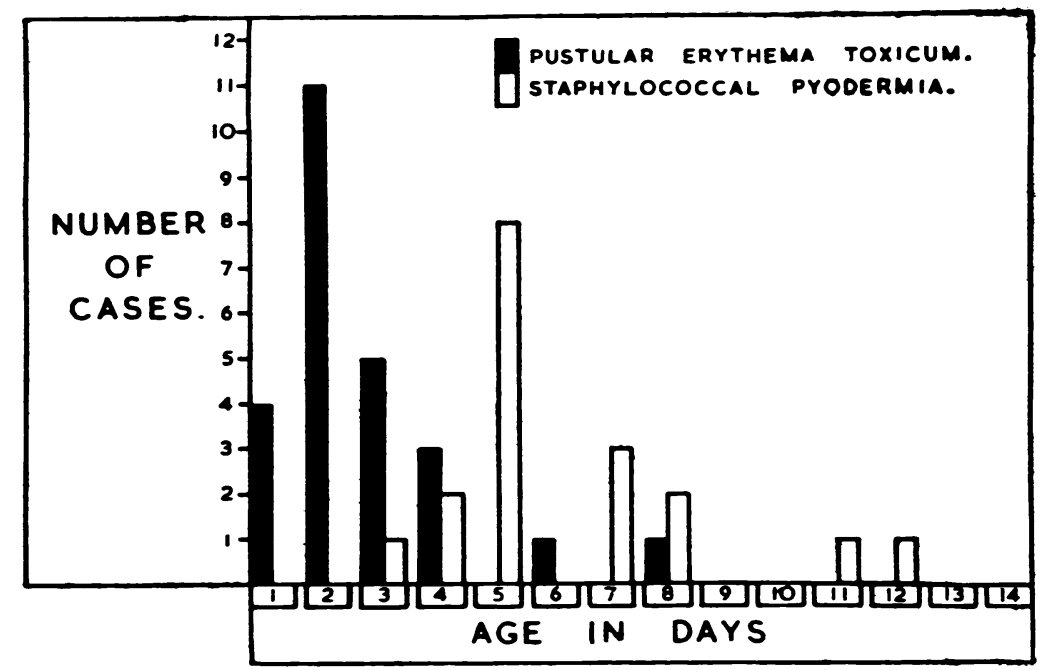

Fig. 4. - Table showing age at first presentation of the rash in 25 consecutive cases of pustular urticaria neonatorum and in 18 consecutive cases of staphylococcal pyodermia seen during the same period. 
best be demonstrated by putting the skin of the affected area on the stretch.

In the pustular type the diagnosis is not always easy. The resemblance to a primary staphylococcal skin infection has already been mentioned, and there is no doubt that in the past we have erroneously treated with antibiotics several cases of pustular urticaria neonatorum.

A pustular rash in the neonatal period may be due to three conditions. In order of frequency during the first week of life, these are: (1) pustular urticaria neonatorum, (2) staphylococcal pyodermia and (3) secondarily infected pustular urticaria neonatorum. The differential diagnosis is of more than academic interest, as it affects such questions as specific treatment and the need for isolation.

In pustular urticaria neonatorum, careful search will frequently reveal the presence of typical urticarial lesions elsewhere on the body. The rash is most common at the age of 2 or 3 days, at a time when staphylococcal infections are rare. After the age of 7 days a pustular rash is very rarely due to urticaria neonatorum. On clinical grounds, therefore, it is often possible to make a correct diagnosis.

In a doubtful case, by far the quickest method of diagnosis (Finlay and Bound, 1952) is to prepare smears from the expressed contents of one of the pustules after puncture by a sterile needle. These smears should be spread thickly and gently, otherwise wholesale fragmentation of leucocytes will occur. This cellular fragility is especially seen if eosinophilic lesions are already in the process of resolving. It is wise to make two smears in each case.

One of the smears is stained by Leishman's method. In the true pustular urticaria neonatorum a striking mass of eosinophil leucocytes, forming the major part of the cellular response, can be seen microscopically (Fig. 5). In the case of a 'primary' staphylococcal pyodermia, such a smear is entirely comprised of neutrophil leucocytes in varying stages of degeneration, while organisms can frequently be seen even in the Leishman film.

Sometimes, however, a mixed picture is seen owing to an eosinophilic lesion having been secondarily invaded by staphylococci. In these, neutrophil leucocytes predominate, but eosinophil leucocytes are still seen in considerable numbers.

When the cellular response indicates the presence of infection the second smear can be stained for organisms by Gram's method, and culture of the pus should also be carried out so that appropriate sensitivity tests may be done.

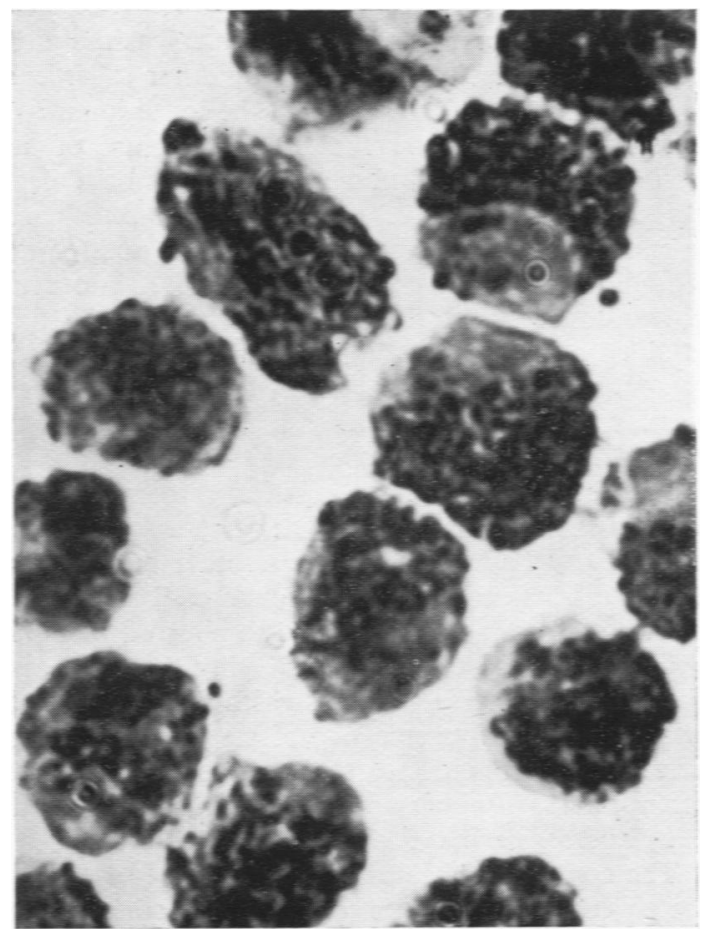

FiG. 5.-Smear from a case of pustular urticaria neonatorum, stained Leishman, showing very numerous eosinophil leucocytes. $(\times 2,800$.)

\section{Treatment}

Provided there is no secondary staphylococcal infection, no treatment is indicated beyond keeping the parts dry with sterile powder. Even the application of a calamine lotion is unnecessary, as there appears to be no associated itching. Complete isolation from other infants is obviated by the correct diagnosis.

If secondary infection is present, antibiotic therapy is indicated.

\section{Aetiology}

Leiner (1912) held the old view that, without exception, the condition was associated with dysfunction of the bowels in the form of dyspepsia, occasional vomiting and frequent bowel action, hence presumably the name 'erythema neonatorum toxicum'. This has not been our experience, and the term 'urticaria neonatorum' is much to be preferred.

The nursing staff are prone to blame over-heating as the main cause, while sensitivity to articles of clothing is often invoked. Mayerhofer and LypoltKrajnović (1927) believed the rash to have numerous causes, especially allergy and the transmission of 'toxins of pregnancy' from the mother to the foetus. Anderson (1950) mentioned that the disorder had 
been attributed also to irritation from oil or soap used for bathing; and to a hypersensitivity to human or cow's milk or to other allergens.

Leiner (1912) pointed out that the condition might possibly represent the first manifestation of an exudative diathesis. Follow-up enquiries have been made regarding our 25 cases of pustular urticaria neonatorum: three could not be traced and, of the remainder, replies were received from 18 mothers. It should be noted that none of the children had attained a greater age than $2 \frac{1}{2}$ years by the time of the enquiry in February, 1953. In no instance had there yet appeared a further individual history of asthma. One infant showed eczema at the age of 5 months. Among near relations, there was an allergic history in six families: two showed both asthma and hay fever, while the other four gave a history of urticaria beyond the neonatal period.

One of our cases, aged 3 days, showed bilateral oedema of the eyelids accompanying the rash, and the stools contained mucus with a little blood, although no pathogenic organisms were found on culture. Examination of the blood showed an eosinophilia. Ephedrine was administered to the baby and all signs quickly subsided.

The common appearance of the rash early in the first week of life rules out the introduction of artificial feeding as a significant cause. Colostrum may be more liable to affect the baby than fully established breast milk. We have enquired with little success into the possibility that drugs or unusual foods taken by the nursing mother might play a part.

In those few cases of pustular urticaria neonatorum encountered with secondary staphylococcal infection so many lesions become infected at once that a blood-borne infection might be suspected. It is more likely, in view of the absence of constitutional disturbance, that the thin walls of the eosinophilic pustules are readily invaded by local spread of staphylococci.

There is obviously scope for much further investigation into the aetiology of this condition. Its cause is at present quite obscure. The consistently transient nature of the rash suggests either that the causative factor is of temporary occurrence or that sensitivity to it is rapidly lost.

\section{Summary}

A clinical description of the urticaria seen in the neonatal period, the so-called erythema toxicum neonatorum, is given and a short reference to the previous literature is included.

The rash is found in two forms, viz. (1) a simple type, and (2) a pustular type.

Observations are made on a series seen in a maternity unit, which included 25 cases of the pustular type. The lesions in the pustular type are shown to be due to a massive local outpouring of eosinophil leucocytes. The resemblance of the pustular type of urticaria neonatorum to staphylococcal pyodermia is stressed and a smear method of rapid diagnosis recommended for doubtful cases.

Occasionally the pustular type becomes secondarily infected.

Uninfected urticaria neonatorum requires no treatment.

Further investigation is urged into the aetiology which is discussed but is at present obscure.

We are grateful to Professor J. L. Henderson for his helpful criticism and advice and to Dr. H. P. Tait for his assistance with the earliest references. Dr. Joyce Morgan has kindly allowed us full access to the notes of the mothers in the Maternity Unit under her care. We wish to thank E. Stride, A.I.B.P., for the photographs and photomicrograph.

\section{REFERENCES}

Anderson, N. A. (1950). In Mitchell-Nelson Textbook of Pediatrics, 5th ed., p. 351. Philadelphia.

Dewees, W. P. (1826). A Treatise on the Physical and Medical Treatment of Children, pp. 359-361. London.

Evanson, R. T. and Maunsell, H. (1836). A Practical Treatise on the Management and Diseases of Children, pp. 194-195. Dublin.

Finlay, H. V. L. and Bound, J. P. (1952). Brit. med. J., 1. 1134.

Hardaway, W. A. (1889). In Keating's Cyclopaedia of the Diseases of Children, vol. 2, p. 18. Edinburgh and London.

Leiner, C. (1912). Über eigenartige Erythemtypen und Dermatitiden des frühen Säuglingsalters, pp. 4-15. Leipzig and Vienna.

Mayerhofer, E. and Lypolt-Krajnović, M. (1927). Z. Kinderheilk., 43,630 .

Salomonsen, L. (1952). In Fanconi and Wallgren's Textbook of Paediatrics, ed. Collis, W. R. F., pp. 239-240. London.

Smellie, W. (1752). A Treatise on the Theory and Practice of Midwifery, vol. 1. p. 439. London.

(1764). A Collection of Preternatural Cases and Observations in Midwifery. (A Treatise on the Theory and Practice of Midwifery, vol. 3), pp. 522-523. London. 